Rasim M. Alguliyev1, Saadat R. Aghayeva ${ }^{2}$

${ }^{1,2}$ Institute of Information Technology of ANAS, Baku, Azerbaijan

1 $\underline{\text { secretary@iit.ab.az, }}{ }^{2}$ seadet.agayeva@gmail.com

\title{
ONLINE MEDIA MONITORING: CURRENT STATE, PROBLEMS AND DEVELOPMENT PROSPECTS
}

\begin{abstract}
The article studies the challenges of internet-media resources monitoring and international experience in this field. The interaction of monitoring and evaluation was presented, the factors facilitating the media monitoring were shown.
\end{abstract}

Keywords: online media, social media, media monitoring, media evaluation.

\section{Introduction}

Information and communication technology (ICT) has penetrated practically all areas, and people have become an integral part of the culture of information. Journalism is one of the priority spheres exposed to the major changes and development as the result of the use of ICT. This development has led to the emergence of new media, in other words, the Internet-media.

In the globalized world, the role and position of the Internet-media is increasing. The abundance of media resources requires their acquisition, sorting, evaluation, and producing the accurate results. The development of web technologies make it possible to define the rate of electronic media and articles, social structure of its readership more accurately and in an objective way [1]. The introduction of such system enables mass media to evaluate its performance and manage its quality.

In modern times, well-known business, governmental and non-governmental organizations monitor their own media organization for the future development of its performance in a positive way. These media groups follow the market trends and analyze business strategies of the competitors. Media monitoring services provide positive results and prospects for the implementation of the above-mentioned objectives.

\section{Why to monitor Mass Media materials?}

Mass Media is one of the most important sources of information and the subject of analysis of officials and experts of trade structures in many organizations. Monitoring and evaluation of newspapers, radio, television, and Internet media resources directs the performance of professional journalists. Today, the efficiency of the public relations department of vast majority of the state, public and business organizations and forming their positive images in the media is an essential condition for their sustainable functioning [2].

Monitoring process includes identification of information sources and their surveillance, data acquisition and storage, selection of the most appropriate data through categorizing and filtering, and finally, analysis of accumulated data [3]. Media organizations monitor own activities to compete with other media groups. Systematic collection of information during the monitoring allows obtaining accurate and reliable information on the topic, evaluating the influence of media groups in the region or in the country, and being aware of information transfer tools. Assessment of the obtained results makes it possible to establish more effective functioning of media resources and optimal decision-making.

Media monitoring around the world was first carried out by "press-clipping" agencies. "Press clipping" is a selection of media publications and visual materials through keywords or other criteria. The first "press-clipping" was founded in London in 1852 by the Polish news agency Romeike. The first Internet media monitoring company, WebClipping, started its operation in 1998 [4]. One of the most professional and leading organizations in this area, FIBEP (International Federation on Press-clipping services), was founded in Paris in 1953 [5]. The organization currently has 92 members from 43 countries. 
Currently, media monitoring conveys not only the search and analysis of information in traditional media, but also working with the Internet resources. It also involves monitoring of social media - forums and blogs on different topics, which does not mean "Media" directly, but can have a significant impact on public opinion and serve as a source of information [6].

Data collection is very important step for the analysis of the materials published in the media. Data collection and monitoring is a reasonable information basis for verified management decision-making [7]. Along with the observation of the materials published in the media on specific topics (e.g., financial markets, information technology, etc.), media monitoring is the result of the observations obtained by keywords.

Regular, initial, competition-oriented, thematic, reputation and other types of Media monitoring are distinguished.

Media monitoring is carried out as follows:

$\checkmark$ observation of close potential source: TV channels, radio stations, periodicals, news sites, etc.;

$\checkmark$ collection of materials;

$\checkmark$ filtration and categorizing;

$\checkmark$ transmission of collected data for further analysis.

\section{Main features of the Media Monitoring}

According to surveys, 9 of the 10 largest US companies annually allocate $\$ 1$ million to the monitoring of the activitis of the competitors. These funds are paid at the expense of proper decisions based on the results achieved from monitoring and analysis of information. Most of monitoring and analysis products are provided with personal or installed autorubricators, including annotators. It proves the given tools to be standards for information and analytical systems. It is important to be able to work with the dictionaries of synonyms and to take into account the morphology of the language in order to use such applications. Without these features the necessary documents can be missed during the search [8].

As a rule, the monitoring of the Internet is used as a supplement to other media monitoring or as a component of other marketing projects.

The monitoring results are presented in the following forms:

- "press clipping": (electronic) scanned articles;

- brief summaries in the required languages;

- statistical report indicating the date, publications, authors, titles, size and pages of the article with the hypertext appropriate to the scanned version of the original text;

- analytical report appropriate to the objective of monitoring.

Media monitoring is applied for:

- operational tracking of negative and potential information risks;

- daily monitoring of positive and negative news in terms of information wars and crisis;

- analysis of the information presented in Mass Media;

- comparison of quality of information about the company in Mass Media;

- comparison of number of positive and negative references;

- evaluation of quality changes in the information of the company compared to its competitors for any period of time.

\section{Monitoring companies and their basic services}

Media monitoring process requires great amount of time and human resources. The companies specialized in media monitoring such as CyberAlert, WPS, Park.ru, Medialogiya, Inteqrum and other news agencies, as well as PR agencies offer media monitoring services to the customers. Media monitoring companies are engaged in the information search in mass media on any criterion. 
Monitoring process. Media monitoring is carried out through various services offered by the monitoring companies. These services are delivered with the use of software that controls the contents of online free news sources, newspapers, magazines, television channels through the automated robots. Monitoring process is implemented as follows: the software reads the text, and then defines the news or social media shares based on each key word of the client, and automatically presents related articles and materials obtained from social media to each client.

Internet-search systems or specialized Mass Media databases (Integrumi, Addictomatic, Alterian, Prnews, Semantic Force, MediaTek, TextAnalyst, TopSOM, IntellSoft Vision, etc.) are often used for monitoring. CyberAlert - best known media monitoring company around the world, monitors 50 thousand online news sources in more than 75 languages every day in 189 countries around the world, more than 50 million blogs in social media, and 100 thousand message boards, forums, complaint sites, more than 200 video sharing websites, including Twitter [9].

Monitoring services include:

- monitoring of all types of Mass Media on the given topics in all countries;

- monitoring of the current situation;

- monitoring of data about media organizations, companies, trademarks, as well as individuals;

- monitoring of past, present, and future events;

- analysis of the current situation;

- analysis of data about media organizations, companies, trademarks, as well as individuals;

- analysis of material published in the media about the client's major competitors;

- identifying statistical characteristics, conclusions and recommendations, and underlying trends.

Monitoring is implemented on certain topics by domestic, regional, international electronic media and news agencies. Monitoring retains the key quotes in the articles giving the customer opportunity to become familiar to all events as soon as possible.

Major monitoring products (as an example of ITAR-TASS monitoring department) may include [7]:

$\checkmark$ daily monitoring;

$\checkmark$ operational on-line monitoring;

$\checkmark$ weekly monitoring with the analytical records;

$\checkmark$ weekly monitoring of foreign Mass Media;

$\checkmark$ monthly monitoring of media organization;

$\checkmark$ monitoring of ordered publications and stories;

$\checkmark$ monitoring of television and radio broadcasting, introducing video and audio stories through information coding (in text format);

$\checkmark$ monitoring of media based on the results of the media events;

$\checkmark$ preparation of the report based on published materials, TV and radio stories;

$\checkmark$ monitoring of publications about the company's activities in the regional media on the areas (summary is provided twice a month and accompanied by an analytical records);

$\checkmark$ summarizing the news on topics of interest to the customer;

$\checkmark$ search for necessary information by keywords.

Media monitoring tools. These tools include computer programs, which collect and sort the data from mass media. There are nearly 100 such programs in the market, but as a rule, all of these programs have thematic rubricators or simple search features without offering medium, or largescale data storage and any quality analysis mechanisms [10]. Text quality analysis is only supposed to assess nuances such as the nature of the relationship between emotions, dangers and objects. Therefore, the human brain that has intuition and is able to gain experience is preferred in this area. Though the estimation speed is not high, the quality is improving. 
Computer program maximum eases the human labor to address intellectual issues. Some applications have options to search over the "intellectual" data sets taking into account morphology and selecting the documents by the lists of key words, their frequency of usage and relative location. Simple content analysis, which is statistical semantic processing, is possible through these applications.

Media monitoring is based on classical factographic analysis methods, including content analysis by quantitative and qualitative information streams:

- analysis of facts;

- evaluation;

- analysis of news topics;

- analysis of news contexts;

- analysis of relations among the contexts;

- analysis of logical expression of news;

- media and statistical analysis;

- analysis of news chronology;

- analysis of bibliographic data (authors, geography, etc.) of news;

- analysis of additional materials (photos, cartoons, etc.) of news [11].

\section{Interaction of Monitoring and Evaluation}

Monitoring, dealing systematic collection and analysis of information, is a precious tool for the management of media group (holding, company, etc.), and it serves as a preliminary stage of the evaluation. Media monitoring allows defining whether how resources are appropriately available and properly used.

Development trends may be reviewed, planning or implementation problems, as well as successful strategies of competitors can be identified through the monitoring and evaluation.

Note that Several monitoring and adjustment procedures are essential before evaluation and reevaluation [12].

Monitoring and evaluation:

$\checkmark$ identify problems and the causes;

$\checkmark$ offer possible solutions to the problems;

$\checkmark$ direct activity of media organization;

$\checkmark$ provide information;

$\checkmark$ encourage to follow the guidance;

$\checkmark$ increase the probable positive growth of the company.

\section{Social media monitoring}

Along with the professional media, social networks play an important role in shaping public opinion in the modern era. In this case, the media, first of all, serves as a means of enlightenment for citizens, on the other hand, as a means of social participation [14]. Unsurprisingly, after the emergence of the Internet, several studies are conducted to reveal the training level of the journalists to dialogue with the audience in Western countries. Studies show that the most journalists do not effort to communicate with the audience. Information technology may affect the development of the media when the possibilities of these technologies would be evaluated and used [15]. 
Overall, data analysis process is carried out as follows (Figure 1):

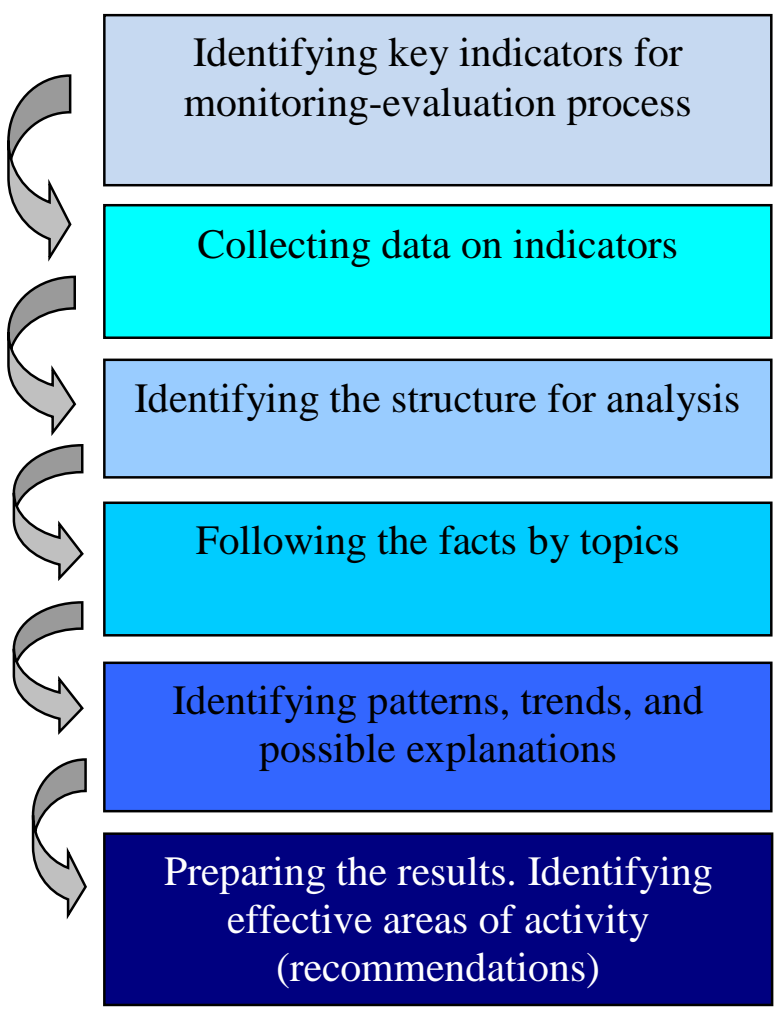

Figure 1. Data analysis process

Transformation of the social media into a new communication means is undeniable. Today, since the social media is one of the best promotional methods, people and companies turn to social networks. Social media is a direct and clear dialogue with the audience, in which website is targeted [16]. Social media means include social networks, blogs, microblogs, forums, and so on. In addition to the monitoring of the Internet-media resources, many media companies monitor social media and review the information spread about them in social media. There are more than 100 different services of monitoring and evaluation of social media. The most well-known companies offering free blog monitoring services are Technorati, Blog Pulse, IceRocket, Google Blogs. The US Technorati, one of the giant companies in the field of web-services and software, reports that 75 million blogs with 1.2 million new daily sharing, which include statements about the company's products and services, are created.

Social media monitoring resolves the following issues [17]:

- consideration of the interests of the audience, defining the needs and aspirations of the people accurately;

- interacting with people;

- spreading information demanded by the audience through social media;

- analyzing the future performance of company.

\section{Status of Media Monitoring in Azerbaijan}

Although there are enough number of Internet media resources, the Internet media is still in the phase of formation [18]. At present, Commission, under the Press Council of Azerbaijan, is operating to control the Internet-media resources. The Commission reviews complaints submitted to the Press Council related to online media resources, monitors national and international trends, and assists to overcome negative consequences in connection with the responsibility of the Internet-media journalist. 
Media Monitoring Center, which deals with media monitoring and analysis in Azerbaijan, "Kalibri" media company monitoring, "A-Vision" media and communications company, and Azerbaijan Media Monitoring Institute offer a wide range of prompt and professional services for the analysis of media and social networking, for the establishment of marketing strategy and tactics in accordance with current trends. The scope of these companies' services includes:

- national media monitoring;

- international media monitoring;

- press monitoring;

- TV broadcasting monitoring;

- radio monitoring;

- social media monitoring;

- monitoring of news agencies;

- monitoring of TV advertising;

- monitoring of street advertising;

- monitoring of advertisements in the press.

The companies prepare monthly statistics of the news submitted to the Internet-media resources. This statistical data expresses the number of used certain keywords of the articles published on the website in figures. Graphical tables are set according to obtained figures. These tables take into account the different forms of one word and indicate only the number. One of the shortcomings of this service is that the content of reports is not analyzed during the preparation of the tables.

Furthermore, various non-governmental organizations periodically monitor the media for various purposes.

\section{Conclusion}

The studies prove the importance of current media monitoring. As the media has a direct impact on public opinion and people's choice, the main goal of media professionals all over the world is to benefit from positive effects of media and work for charitable purposes. To this end, Mass Media may promote the reputation of the site by media monitoring and making rational decisions to set the right strategies. The results of the studies define the following problems of online media monitoring:

1. High-cost monitoring services: media groups applying to monitoring companies may face high prices. The offered prices challenge the use of media monitoring services by smaller companies.

2. Inconsistency of the results. Sometimes, the results of several monitoring companies do not coincide.

3. Automated tools: these cannot define positive, negative or neutral information.

Analysis shows that the resolution of existing media monitoring problems requires conceptual and institutional approach. It may also be significant for the main objectives of the national security as the identification of freedom of information, the public sentiment, public opinion, as well as for the detection of threats.

In addition, lack of application of new technologies in the monitoring, inconsistencies during the monitoring procedure, the absence of confidence as a result of deregulation of this area, the deficiency of necessary culture, nonexistence of intelligent monitoring and analysis, and the lack of national statistics mechanisms for the Internet resources are the obstacles for the development of media monitoring.

Thus, media monitoring enables:

- overall assessment of the situation in the field of information;

- revealing opportunities for improving the performance of information field through media and analytics; 
- developing information policy by considering general media image and situation in this field;

- scheduling several measures to be taken in the field of public relations based on the experience of the competitors and related companies;

- predicting the events and actions of competitors;

- optimizing the work with publications and journalists (promoting database of journalists, journalists' rating, publications' rating, mass media databases).

It can be noted that the monitoring of only the Internet-media resources does not provide the evaluation of the entire media processes. Thus, the steady increase in the number of media sites does not mean complete decline of traditional media.

\section{References}

1. O. Gunduz. Information technologies for journalists. Baku, 2009, p.101.

2. Phillips D. PR in the Internet. trl. from Eng. by I.Gavrilov. M. Fair-Press, 2004, p.320.

3. Media Monitoring Service, http://pages.rediff.com/media-monitoring-service/305165

4. http://www.cision.com/us/pr-software/media-monitoring/online-media-monitoring

5. http://www.fibep.info

6. Huridocs, Media monitoring, information scanning and Intelligence, 2009, $33 \mathrm{p}$.

7. Lysenko A.G., Fateev E.A. Journalistic skills. An analysis of media materials, Moscow, p.25.

8. Oparin A., Media monitoring and analysis systems, December 16, 2003, http://www.newart.ru/oparin/smi_oparin.htm

9. CyberAlert, Inc. Media Monitoring: The Complete Guide, 2010, p.26.

10. Daniels M., Jeffrey A., International Media Analysis Made Simple, Institute for Public Relations, 2012. 34 p.

11. Media analysis, http://www.public.ru/analytics

12. Shapiro J. Monitoring and Evaluation, South Africa, Johannesburg 2001, 51 p.

13. Institute of Information Freedom Development, Methods of monitoring the sites of nonprofit organizations, St. Petersburg, 2011, p.69.

14. Kaschesky M., Sobkowicz P., Lobato J.M.H. et all. Bringing Representativeness into Social Media Monitoring and Analysis. 46th Hawaii International Conference on System Sciences, 2013, 10 p.

15. Alguliyev R.M., Mahmudov R.Sh. Emergence and development of the Internet media: key trends and characteristics. Express-information, Information Society series, Baku, "Information Technology" Publishing House, 2012, p.58.

16. http://www.snce.ru/ru

17. http://www.cosida.com/news/2014/10/7/imported_1007141327.aspx

18. Valiyev S., Babayev Z. Internet journalism in Azerbaijan: history and prospects. B., 2007, p. 247. 